\title{
A SEARCH FOR PERIODICITIES IN THE LIGHT VARIATION OF SOME SEYFERT GALAXIES
}

\author{
S.M. Bisch, M.G. Pastoriza, H. Dottori, I. Busko* \\ Instituto de Fisica, UFRGS \\ Av. Bento Gonçalves, 9500 \\ 90.000 Porto Alegre, RS Brasil \\ * Laboratório Nacional de Astrofísica, CNPq
}

ABSTRACT. Penfold's UBV observations of the Nuclei of the Seyfert galaxies NGC 1566, NGC 3227, NGC 3783, NGC 6814, NGC 7469, MK 50 and IC 4329A are examined to look for a possible periodicity in the variation of the magnitudes and colors. A method based on the fitting of sinusoidals and the calculation of the multiple correlation coefficient adequated to the analysis of observations unequally spaced in time is used to analyse the data. Four of the seven galaxies studied show a high probability of having periodic component in the variation. Recent UBV observations were used to test the components in two of them.

\section{INTRODUCTION}

Systematic UBV observations of the nuclei of Seyfert galaxies were carried out by Lyutyi (1973, 1977, 1979), Penston et al. (1974) and Penfold.(1979).

The possible existence of periodic components in the visual light variation of some Seyfert galaxies was suggested by Lyutyi and Penfold. Lyutyi suggests the existence of "slow" and "rapid" components superimposed in the brightness variation.

An analysis using a period search technique has been done for the optical variability of NGC 4151 by Belokon et al. (1978) and Lyutyi and Okyanskii (1981). In the first paper, photoelectrical observations covering the period 1970-1973 were analyzed and a 130 day period was found for the "rapid" component. In the second a light curve covering more than 20 years composed by photographic (1958-1976) and photoelectrical (1967-1980) observations were analyzed. The presence of a quasiperiodic component of 126 day was confirmed and a multicomponent structure was suggested to explain the slow variation. A more extense set of photographic and photoelectrical observations of NGC 4151, 
covering several decades, was analyzed by Pacholczyk et al. (1983) using a power spectrum procedure. Contrary to the previous results, they concluded that there was no evidence of periodicity in the light variations of this galaxy.

The controversy on this subject, as well as its importance for the formulation of nuclei models, induced us to analyse the light curves of other seyfert galaxies. In this paper we present the analysis of Penfold's (1979) UBV observations of the Seyfert galaxies NGC 1566, NGC 3227 , NGC 3783, NGC 6814, NGC 7469, IC 4329A and Mk 50 by means of a method of periodicity analysis based on the fitting of sinusoidals and the calculation of the multiple correlation coefficient. Recent UBV observations were carried out in order to test the estimated periods.

The Observations

From Penfold's observations in the period of 1974-1976, we selected the galaxies reported as variables in the $U$. B. V magnitude and $U-B, B-V$ colors, in the 14.3" diaphragm. From 1981 to 1983 we added $U, B, V$ observations of NGC 6814 and NGC 7469 carried out with the $1.6 \mathrm{mt}$ telescope, 14" diaphragm, in the Laboratório Nacional de Astrofisica (LNA). The observed values are 1 isted in table 1 .

Table 1. LNA Observations with 14" Diaphragm

\begin{tabular}{ccccccc}
\hline NGC & Julian Date & $\mathrm{U}$ & $\mathrm{B}$ & $\mathrm{V}$ & $\mathrm{U}-\mathrm{B}$ & $\mathrm{B}-\mathrm{V}$ \\
\hline \multirow{2}{*}{6814} & 2445079 & 14.20 & 14.41 & 13.52 & -0.24 & 0.89 \\
& 2445207 & 14.43 & 14.52 & 13.65 & -0.10 & 0.87 \\
& 2445554 & 14.23 & 14.50 & 13.61 & -0.27 & 0.89 \\
7469 & 2445591 & 14.29 & 14.58 & 13.65 & -0.29 & 0.95 \\
& 2445206 & 12.85 & 13.54 & 13.10 & -0.68 & 0.43 \\
& 2445554 & 12.77 & 13.55 & 13.10 & -0.78 & 0.44 \\
& 2445591 & 12.98 & 13.62 & 13.55 & -0.64 & 0.47 \\
\hline
\end{tabular}

The Method and the Analysis Scheme

The method adopted for the search of periodicity, hereafter called MCC, is based on the calculation of the multiple correlation coefficient $R$, which is defined by

$$
R^{2}=\frac{\sum_{i=1}^{N}\left(\hat{y}_{i}-\bar{y}\right)^{2}}{i=1\left(y_{i}-\bar{y}\right)^{2}}
$$

where $\mathrm{N}$ is the number of observations, $y_{j}$ is the observed magnitude at an instant $t_{i}, \bar{y}$ is the mean value of the 
observations and $\hat{y}_{i}$ is the value of the fitted function at the same instant $t_{i}$. The best fitting corresponds to the greatest value of ${ }^{1} R^{2}$. Initially, the $R^{2}$ coefficient was calculated for various frequencies corresponding to periods from 10 to 10000 days, with a 0,0001 day $^{-1}$ step using a sinusoidal plus a constant as fitting function. The plot of $R^{2}$ versus frequency, hereafter called the spectrum, presents spikes on those frequencies corresponding to periods associated with any real periodic variation.

The uncertainty in the value of $R^{2}$ at any spike due to the uncertainty in the data can be calculated by error propagation:

$$
\sigma_{R^{2}}^{2}=\frac{4\left[R^{2}-\left(R^{2}\right)^{2}\right]}{\left.N=1 \sum_{i}-\bar{y}\right)^{2}},
$$

where $\sigma_{i}$ is the uncertainty in each observation.

$A$ test can be made in order to check the probability that given spike occurred by chance, doing the hypothesis that the variations of the data are purely randomic (with gaussian distribution). Under this hypothesis, the probability of obtaining $\mathrm{R}^{2}$ greater than a certain value $A$ is given by

$$
\begin{aligned}
\operatorname{prob}\left(R^{2} \geq A\right)= & (1-A)^{n / 2}\left[1+\frac{1}{1 !} \frac{n}{2} A+\frac{1}{2 !} \frac{n}{2}\right. \\
& \left(\frac{n}{2}+1\right) A^{2}+\cdots+\frac{1}{\left(\frac{m}{2}-1\right) !} \\
& \frac{n}{2}\left(\frac{n}{2}+1\right)\left(\frac{n}{2}+2\right) \ldots \\
& \left(\frac{n}{2}+\frac{m}{2}-2\right) A^{\left.\frac{m}{2}-1\right] .}
\end{aligned}
$$

This expression is valid when $m$, the number of parameters of the fitting function minus one, is even. The parameter $n$ is defined as

$$
n=N-m-1 \text {. }
$$

In order to search for other periodic components, besides the one already selected, it is possible to filter out the initial fitting function and repeat the analysis process until the height of the salient spikes minus the uncertainty approximates the level of the noise in the spectrum.

A characteristic of the method is the appearance of spurious spikes (aliases) in the spectrum due to the fact 
that the observations are regulated by the dark moon and the solar annual periods. The two principal aliases related to a real frequency $\nu_{0}$ are given by

$$
\nu \simeq\left|v_{0} \pm \frac{1}{\Delta t}\right| \text {, }
$$

where $\Delta t$ is the approximately constant spacement between successive observations. In order to distinguish between spurius and real spikes, we subdivided the observations into shorten time intervals, and repeated the calculation of the spectrum.

\section{Results}

It is not found a single behavior for the variation of the studied Seyfert nuclei. Whereas Mk 50 is clearly represented by a one period light curve of 450 days (Figure 1 ) of high probability (Figure 2) NGC 3227, NGC 7469, and IC 4329A are fitted by the superposition of a slow and a fast component. The light curve of the rest of the objects are not clearly defined by periodic components, showing a more chaotic light variation, as found for NGC 4151 by Pacholczyk et al. (1983).

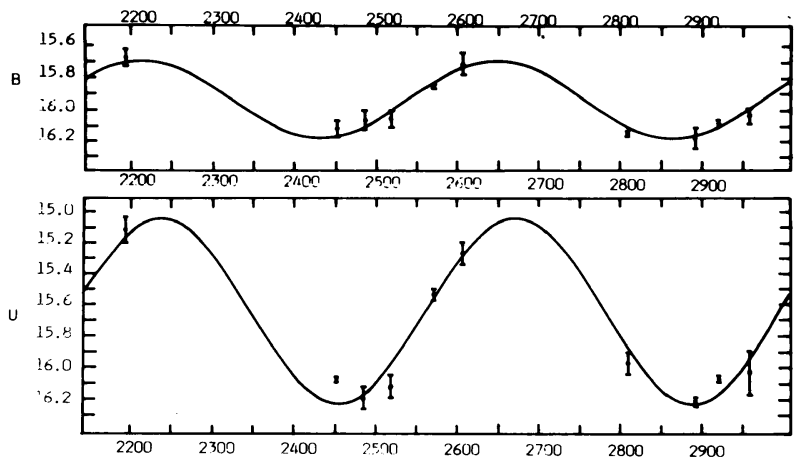

Figure 1. Mk 50 fitting with period of 430 days, Julian Date $(2440000+)$
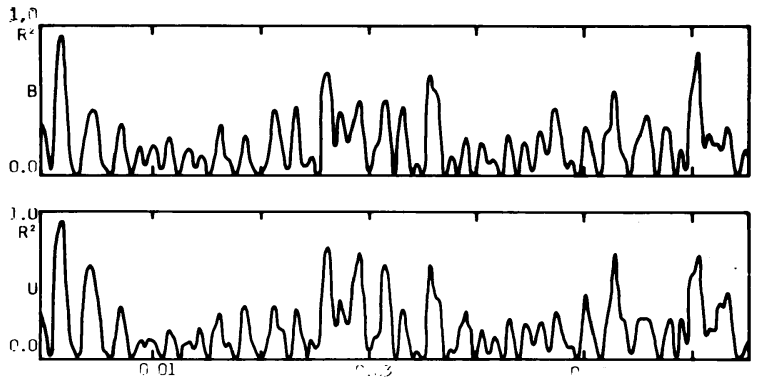

Figure 2. Mk 50 spectra of frequencies. The most salient spike is at $\nu=0.0023$ (430 days). 
For NGC 7469 the Figures $3-a$ and $3-b$ show that Penfold's and Penfold's+LNA data are fitted by the same couple of periods and phase. When analyzed NGC 6814, the observations of Penfold+LNA do not repeat the periods found for the Penfold's one. It is important to emphasize that the same periods are found for the three colors. The amplitude of the slow component decreases from $U$ to $V$ color.

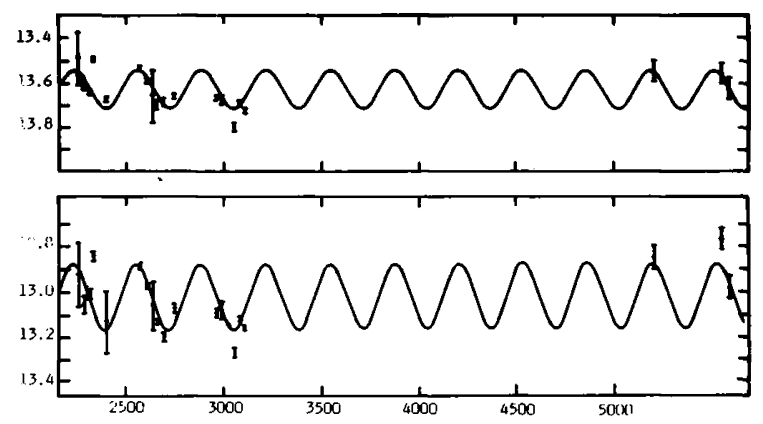

Figure 3.a NGC 7469 fitting of Penfold's data with periods 3000 and 128 days. Julian Date $(2440000+$ )

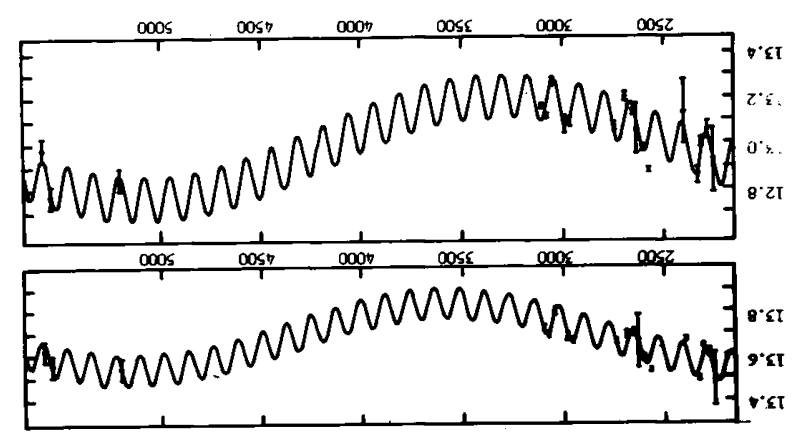

Figure 3-b. NGC 7469 fitting of Penfold's+LNA data with periods 3000 and 128 days. Julian Date (2440000+)

A summary of the results is presented in table 2 . 
Table 2. Parameters of the Fitting Function

\begin{tabular}{|c|c|c|c|c|c|}
\hline \multirow{3}{*}{$\begin{array}{l}\text { Galaxy } \\
\text { Mk50 }\end{array}$} & \multirow{3}{*}{$\begin{array}{c}\text { Estimated } \\
\text { Period(s) } \\
\text { (Day) }\end{array}$} & \multicolumn{2}{|c|}{$\begin{array}{l}\text { Amplitude } \\
\text { (Mag) }\end{array}$} & \multicolumn{2}{|c|}{$\begin{array}{l}\text { Fitting } \\
\text { Function }\end{array}$} \\
\hline & & $\Delta U$ & $\Delta \mathrm{B}$ & & \\
\hline & & 1.18 & 0.48 & 0.952 & 0.0024 \\
\hline NGC $\quad 3227$ & 1000 & 0.81 & - & 0.970 & 0.0004 \\
\hline NGC 7469 & $\begin{array}{r}150 \\
3000 \\
125\end{array}$ & $\begin{array}{l}0.47 \\
0.32 \\
0.23\end{array}$ & $\begin{array}{l}- \\
0.17 \\
0.14\end{array}$ & 0.863 & 0.01 \\
\hline IC $4329 \mathrm{~A}$ & $\begin{array}{r}10000 \\
500\end{array}$ & $\begin{array}{l}1.64 \\
0.27\end{array}$ & $\begin{array}{l}0.42 \\
0.19\end{array}$ & 0.973 & 0.0001 \\
\hline
\end{tabular}

\section{Conclusion}

The method of MCC for the analysis of periodicity shows that the light curve of 7 seyfert nuclei in the UBV colors do not present a unique behavior. One of the galaxies Mk50 shows a well defined single period. Three of them can be described by the superposition of a slow and rapid periodic component. The remaining galaxies show a more chaotic light variation.

\section{References}

Belokon, E.T., Babadzajanz, M.K., Lyutyi, V.M.: 1978, Astron. Astrophys. Suppl. 31, 383.

Lyutyi, V.M.: 1973, Sov. Astron. 16, 763.

Lyutyi, V.M.: 1977, Sov. Astron. 21, 655.

Lyutyi, V.M.: 1979, Sov. Astron. 23, 518.

Lyutyi, V.M., Oknyanskii, V.L.: 1981, Sov. Astron. Lett. 7, 364 .

Pochalczyk, A.G., Lubart, N.D., Penning, W.R., Ferguson, D.H., Turnshek, D.: 1983, Astrophys. Lett. 23, 225.

Penfold, J.E.: 1979, Mon. Not. R. Astr. Soc. 186, 297.

Penston, M.V., Penston, M.J., Selmes, R.A., Becklin, E.E., Neugebauer', G.: 1974 , Mont. Not. R. Astr. Soc. 169, 357.

Ácknowledgement

The authors are thankful to the Brazilian Institutions CNPq, CAPES, and FINEP for the partial support of this work. 